\title{
An attitudinal trust recommendation mechanism to balance consensus and harmony in group decision making
}

\author{
Jian Wu, Senior Member, IEEE, Xue Li, Francisco Chiclana, and Ronald R. Yager, Fellow, IEEE
}

\begin{abstract}
This article puts forward a trust based framework for building a recommendation mechanism for consensus in group decision making with interval-valued intuitionistic fuzzy information. To do that, it first presents an attitudinal trust model where experts assign trust weights to others considering the concept of attitude of the group. This approach allows for the implementation of the group attitude in a continuous scale ranging from a pessimistic attitude to an indifferent attitude. Thus, it can express the continuous trust status, and consequently it generalizes the traditional simplified trust model: 'trusting' and 'distrusting'. In particular, three typical policies are defined as: 'extreme trust policy', 'bounded trust policy' and 'indifferent trust policy'. Secondly, the attitudinal trust induced recommendation mechanism is established by a reasonable rule: the closer the experts, the higher their trust degree. This can guarantee that the consensus level of the inconsistent expert is increased after adopting the recommended advices. In addition to group consensus, experts envisage to keep their original opinions as much as possible. A harmony degree (HD) is defined to determine the extent of the difference between an original opinion and the corresponding revised opinion after adopting the recommended advices. Combining the HD index and the consensus index, a sensitivity analysis with attitudinal parameter is proposed to verify the rationality of the proposed attitudinal trust recommendation mechanism. In practice this will facilitate the inconsistent experts to achieve a balance between consensus degree and harmony degree by selecting an appropriate attitudinal parameter.
\end{abstract}

Index Terms-Group decision making; Consensus; Intervalvalued intuitionistic fuzzy sets; Trust; Attitude

\section{INTRODUCTION}

$\mathbf{I}$ $\mathrm{N}$ group decision making (GDM) problems, each expert expresses his/her individual preference on a set of alternative under multiple criteria. Then, these individual preferences are aggregated into a collective one to derive a common solution. However, In the actual process, it is difficult or even impossible for an expert to give an accurate evaluation information due to the ambiguity of decision making problem. The theory of fuzzy sets (FSs) proposed by Zadeh [51] is a powerful tool to deal with ambiguity, whose basic component is only a membership function. After that, Atanassov [4] proposed the definition of intuitionistic fuzzy sets in which components are membership functions and non-membership functions, and

Jian $\mathrm{Wu}$ and Xue Li are with the School of Economics and Management, Shanghai Maritime University, Shanghai 2013026, China (jyajian@163.com; leexue10028@163.com). Francisco Chiclana is with the Institute of Artificial Intelligence (IAI), Faculty of Computing, Engineering and Media, De Montfort University, Leicester, UK (chiclana@dmu.ac.uk) and with the Dept. of Computer Science and Artificial Intelligence University of Granada, Spain. Ronald R. Yager is with the Machine Intelligence Institute, Iona College, New Rochelle, NY10801 USA (yager@panix.com). then it is an extension of the fuzzy sets. However, due to the complexity of decision problem and the limitations of the knowledge, experts may not be easy to express exact values for the membership and nonmembership degrees of an element to a given set [18]. In this case, interval number are rather than crisp values to express the membership and nonmembership degrees under the environment with uncertainty, imprecision or vagueness. Therefore, Atanassov and Gargov[3] introduced the concept of interval intuitionistic fuzzy sets in which the membership and non-membership degrees are expressed by intervals. A great number of work has enriched this field of decision making with interval intuitionistic fuzzy sets in [18], [33], [38], [44].

However, due to experts' different background and knowledge level of the decision problem, there usually exists inconsistency (disagreement) [1], [31], [38]. Therefore, it is preferable that the group achieves agreement before making a decision, i.e. before individual preferences are aggregated [6], [15], [28], [30], [42]. A consensus reaching process (CRP) is regarded as an effective approach to reduce or eliminate inconsistency in GDM [10], [11], [26]. It usually involves a recommendation mechanism to generate advices for the inconsistent experts [25], [27], [52], [53] to become aware of how to modify their inconsistent preferences to increase the consensus level.

How to construct recommendation mechanisms to generate advices is therefore a key issue in GDM consensus. In a 'traditional' recommendation mechanism, the preference advice is usually generated as the arithmetic average of all individual preferences [29], [43], [45], [55]. However, this approach cannot generate personalized advice; additionally, it neglects the trust relationship among group experts, which has been regarded as an important resource to induce the group to reach consensus in GDM [14]. Examples of the use of trust in GDM consensus include: trust propagation based recommendation[32], [39]; trust based aggregation of individual opinions [5], [8], [36]; trust based estimation of incomplete information [37]; and trust based group network clustering [2]. Obviously, these research works draw on the general veracious claim of individual experts possessing different trust degrees with others in the interaction process of GDM, which is used here to investigate a more reasonable recommendation mechanism to allow the inconsistent experts to revisit their preferences according to the advice from the other experts they trust. It is worth noting that few scholars pay attention to the research to this issue. 
Liu et al.in [24] proposed a trust induced recommendation mechanism for GDM in which the experts are divided into two sets: the trusted experts and the distrusted experts, who are above and below a fixed threshold, respectively. Then, the inconsistent expert can adopt the recommended advice from his/her trusted experts and neglect those coming from the distrusted ones. However, this trust classification is too absolute because it relies on a binary relation use of 'trusting' and 'distrusting'. In most case, experts do not merely express the terms 'trusting'/'distrusting', but rather quantify the level of trust on someone with words such as 'high' or 'low' [40]. We believe that the binary use of trust [24] is a rather simplistic trust mechanism because it does not consider the behaviour of experts, and therefore, it is not rich enough to capture all the information contained in realistic trust relationship. Recently, Yager and Alajlan [48] presented a very interesting idea for the CRP stopping policy that can reflect the attitude of a group. Inspired on this idea, this article aims to propose a recommendation mechanism driven by a general the attitudinal trust model that will generate trust recommended advices based on the reasonable trust policy: the closer the experts' opinions are, the higher their trust degree will be. In particular, this article defines three typical trust policies: 'extreme trust policy'; 'bounded trust policy'; and 'indifferent trust policy'. Finally, this attitudinal trust induced recommendation mechanism can guarantee that the consensus level of the inconsistent expert will increase after adopting the recommended advices.

In addition to group consensus, experts also aim at keeping their original opinions in order to maintain their group independence. The term 'group independence' can be captured with what we call the 'harmony degree' (HD), which will measure the extent of the difference between the original opinion and the revised opinion after adopting the group advices [41]. The bigger the HD index is, the lower the adjustment cost of the inconsistent experts will be [12], [13], [40]. Furthermore, the HD index is proved to be bigger than the average degree of conformity of an expert in the group (ACD index), which means that the inconsistent expert prefers to keep their opinion rather than modify it towards the group opinion in GDM consensus. Combining the HD and ACD indices, a sensitivity analysis with attitudinal parameter is proposed to verify the rationality of the proposed attitudinal trust recommendation mechanism. This way, the inconsistent experts can select an appropriate attitudinal parameter to achieve a desired balance between consensus and harmony.

The rest of the article is set out as follows: Section II introduces the definition of interval-valued intuitionistic fuzzy sets, which are used to represent experts preferences. Section III defines the concept of the attitudinal trust degree (ATD), and then proposes an ATD induced recommendation mechanism for consensus in GDM. The definition of harmony degree (HD) is elaborated and justified in Section IV, which is followed by a rationality analysis of the attitudinal trust recommendation mechanism. Section $\mathrm{V}$ proposes a selection process of alternative after group experts reach consensus. Section VI introduces the process of consensus based on attitudinal trust induction recommendation mechanism. Section VII draws the conclusions.

\section{Preliminaries}

Atanassov and Gargov's [3] concept of interval-valued intuitionistic fuzzy sets (IVFS), which are characterized by a membership and a nonmembership functions, permits to process hesitancy information in decision making problems [19], [20], [22], [33], [34], [50], [56].

Definition 1 (Interval-Valued Intuitionistic Fuzzy Set (IVIFS)). "Let INT $([0,1])$ be the set of all closed subintervals of the unit interval and $X$ be a universe of discourse. An interval-valued IFS (IVIFS) A over $X$ is given as:

$$
A=\left\{\left\langle x, \tilde{\mu}_{A}(x), \tilde{\nu}_{A}(x)\right\rangle \mid x \in X\right\}
$$

where $\tilde{\mu}_{A}(x), \tilde{\nu}_{A}(x) \in \operatorname{INT}([0,1])$, represent the membership and the non-membership degrees of the element $x$ to the set $A$ subject to the constraint $0 \leq \sup \tilde{\mu}_{A}(x)+\sup \tilde{\nu}_{A}(x) \leq$ $1, \forall x \in X$.

Denoting by $\tilde{\mu}_{A L}(x), \tilde{\mu}_{A U}(x)$ and $\tilde{\nu}_{A L}(x), \tilde{\nu}_{A U}(x)$ the lower and upper end points of $\tilde{\mu}_{A}(x)$ and $\tilde{\nu}_{A}(x)$, respectively, an IVIFS can be represented as $A=$ $\left\{\left\langle x,\left[\tilde{\mu}_{A L}(x), \tilde{\mu}_{A U}(x)\right],\left[\tilde{\nu}_{A L}(x), \tilde{\nu}_{A U}(x)\right]\right\rangle \mid x \in X: 0 \leq\right.$ $\left.\left.\tilde{\mu}_{A U}(x)+\tilde{\nu}_{A U}(x)\right) \leq 1, \tilde{\mu}_{A L}(x) \wedge \tilde{\nu}_{A L}(x) \geq 0\right\}$. The hesitancy degree function of an IVIFS is $\tilde{\pi}_{A}(x)=\left[1-\tilde{\mu}_{A U}(x)-\right.$ $\left.\tilde{\nu}_{A U}(x), 1-\tilde{\mu}_{A L}(x)-\tilde{\nu}_{A L}(x)\right] . "$

To make a ranking order of IVIFNs, $\mathrm{Xu}$ and Chen proposed in [44] the following score degree and accuracy degree functions:

Definition 2. Let $\widetilde{\alpha}=\left(\left[\mu^{-}, \mu^{+}\right],\left[\nu^{-}, \nu^{+}\right]\right)$be an IVIFN. The score degree and accuracy degree functions of $\widetilde{\alpha}$ are represented, respectively, by

$$
\widetilde{S}_{X C}(\widetilde{\alpha})=\frac{\mu^{-}+\mu^{+}-\nu^{-}-\nu^{+}}{2}
$$

and

$$
\widetilde{A}_{X C}(\widetilde{\alpha})=\frac{\mu^{-}+\mu^{+}+\nu^{-}+\nu^{+}}{2} .
$$

The above score degree and accuracy degree by $\mathrm{Xu}$ and Chen [44] are used to develop the following IVIFNs two level ranking method:

Definition 3. Given two IVIFNs $\widetilde{\alpha}_{1}=\left(\left[\mu_{1}^{-}, \mu_{1}^{+}\right],\left[\nu_{1}^{-}, \nu_{1}^{+}\right]\right)$ and $\widetilde{\alpha}_{2}=\left(\left[\mu_{2}^{-}, \mu_{2}^{+}\right],\left[\nu_{2}^{-}, \nu_{2}^{+}\right]\right)$, the following ordering relation can be established:

(1) If $\widetilde{S}_{X C}\left(\widetilde{\alpha}_{1}\right)<\widetilde{S}_{X C}\left(\widetilde{\alpha}_{2}\right)$ then $\widetilde{\alpha}_{1}<\widetilde{\alpha}_{2}$.

(2) If $\widetilde{S}_{X C}\left(\widetilde{\alpha}_{1}\right)=\widetilde{S}_{X C}\left(\widetilde{\alpha}_{2}\right)$ :

(i) If $\widetilde{A}_{X C}\left(\widetilde{\alpha}_{1}\right)<\widetilde{A}_{X C}\left(\widetilde{\alpha}_{2}\right)$ then $\widetilde{\alpha}_{1}<\widetilde{\alpha}_{2}$.

(ii) If $\widetilde{A}_{X C}\left(\widetilde{\alpha}_{1}\right)=\widetilde{A}_{X C}\left(\widetilde{\alpha}_{2}\right)$ then $\widetilde{\alpha}_{1}=\widetilde{\alpha}_{2}$.

The intuitionistic fuzzy set (IFS) and the interval-valued fuzzy set (IVFS) are mathematically equivalent [9], and so the intuitionistic fuzzy decision matrix (IFDM) and the intervalvalued fuzzy decision matrix (IVFDM) are [35]. This result was eventually exploited by $\mathrm{Wu}$ et al. in [38] to derive the correct theoretical formulation of the multiplicative transitivity property. Accordingly, IFSs and IVFSs are completely 
equivalent in MCDM resolution processes and, as a result, hesitation and uncertainty can be unified. The concept of interval-valued intuitionistic fuzzy decision matrix (IVIFDM) derives from the the definition of interval-valued fuzzy number as a generalisation of the concept of fuzzy decision matrix [17], [38]. Indeed, a matrix $A=\left(\tilde{a}_{i j}\right)_{m \times n}$ with IVIFN elements $\tilde{a}_{i j}$ will be called an IVIFDM. As consequence of the isomorphism between intuitionists and interval-valued fuzzy sets, the operational laws of the latter will be implemented herein.

\section{ATtitude BASED TRUST RECOMMENDATION MECHANISM FOR GDM CONSENSUS}

As aforementioned, group of experts may have inconsistency among their opinions. Therefore, it is advisable for a group of experts to reach consensus before implementing the aggregation step of the GDM process. The recommendation mechanism is very useful to generate advice for the inconsistent experts to increase the group consensus level. Usually, the recommended advice is generated using the arithmetic average of all individual opinions [21], [29], [43], [45], [55]. However, this approach does not take into account the trust relationship within a group of experts, and then the inconsistent experts are forced to adopt the recommended advices without considering whether they trust them or not [54]. Recently, Liu et al. [24] used trust to develop a recommendation mechanism for GDM consensus where the trust relationship is reduced to a binary relation: 'trusting' and 'distrusting'. However, in most case, experts do not merely express the terms 'trusting' and 'distrusting', and they also quantify the level of trust on someone with words such as 'high' or 'low' [40]. To resolve this problem, this article aims to propose a general trust model based on the concept of attitude and the reasonable trust policy: the closer the experts' opinions are, the higher their trust degree will be. Then, an attitude based trust recommendation mechanism for group consensus under interval-valued intuitionistic fuzzy sets is proposed (see Fig. 1). It includes the following five steps: (1) Calculating the consensus degree; (2) Identification of the inconsistency experts; (3) Constructing the trust matrix and defining the concept of attitudinal trust function; (4) Trust induced feedback mechanism and feasibility analysis; (5) Selection process.

\section{A. Consensus degree with interval-valued intuitionistic fuzzy sets}

The interaction of group consensus is actually a dynamic group discussion process [29]. In order to achieve group consensus, the inconsistent experts must be identified before adopting the recommendation advices. This paper constructs the consensus evaluation index, and defines the consensus degree (CD) of each expert, $\left\{e_{1}, \ldots, e_{k}\right\}$, based on the proximity index between opinions.

Let $\tilde{R}^{h}=\left(\tilde{r}_{i j}^{h}\right)_{m \times n}$ and $\tilde{R}^{l}=\left(\tilde{r}_{i j}^{l}\right)_{m \times n}$ be the intervalvalued fuzzy evaluation matrices provided by expert $e_{h}$ and expert $e_{l}$, respectively, on a set of alternatives $\left\{x_{1}, \ldots, x_{m}\right\}$ based on a set of attributes $\left\{c_{1}, \ldots, c_{n}\right\}$.
Level 1. Consensus degree on evaluation element level. For a pair of experts, $e_{h}$ and $e_{l}$, their consensus degree on the alternative $x_{i}$ for attribute $c_{j}$ is:

$$
\begin{gathered}
C E_{i j}\left(\tilde{R}^{h}, \tilde{R}^{l}\right)=1-d\left(\tilde{r}_{i j}^{h}, \tilde{r}_{i j}^{l}\right) \\
=1-\frac{\left|\mu_{i j}^{h-}-\mu_{i j}^{l-}\right|+\left|\mu_{i j}^{h+}-\mu_{i j}^{l+}\right|+\left|v_{i j}^{h-}-v_{i j}^{l-}\right|+\left|v_{i j}^{h+}-v_{i j}^{l+}\right|}{4}
\end{gathered}
$$

It is $C E_{i j}\left(\tilde{R}^{h}, \tilde{R}^{l}\right) \in[0,1] \forall i, j, h, l$, with the maximum value 1 obtained only when both experts have the same preference values on the alternative $x_{i}$ for the considered attribute $c_{j}$.

For a given expert, $e_{h}$, his/her average degree of consensus with the group on alternative $x_{i}$ for attribute $c_{j}$ is:

$$
A C E_{i j}^{h}=\frac{1}{k-1} \sum_{l=1, h \neq l}^{k} C E_{i j}\left(\tilde{R}^{h}, \tilde{R}^{l}\right)
$$

Again, $A C E_{i j}^{h} \in[0,1] \forall i, j, h$, and the maximum value 1 implies that all experts in the group have the same preference value on the alternative $x_{i}$ for the considered attribute $c_{j}$. In general, the greater the value of $A C E_{i j}^{h}$, the higher the consensus of an expert with the group on alternative $x_{i}$ for attribute $c_{j}$.

Level 2. The consensus degree on alternative $x_{i}$ for a pair of experts, $e_{h}$ and $e_{l}$ is:

$$
C A_{i}\left(\tilde{R}^{h}, \tilde{R}^{l}\right)=\frac{1}{n} \sum_{j=1}^{n} C E_{i j}\left(\tilde{R}^{h}, \tilde{R}^{l}\right)
$$

For a given expert, $e_{h}$, his/her average consensus degree with the group on alternative $x_{i}$ is:

$$
A C A_{i}^{h}=\frac{1}{k-1} \sum_{l=1, h \neq l}^{k} C A_{i}\left(\tilde{R}^{h}, \tilde{R}^{l}\right)
$$

Notice that it is $A C A_{i}^{h}=\frac{1}{n} \sum_{j=1}^{n} A C E_{i j}^{h}$.

Level 3. The consensus degree on the decision matrix. For a pair of experts, $e_{h}$ and $e_{l}$, their consensus degree on their decision matrices is:

$$
C D\left(\tilde{R}^{h}, \tilde{R}^{l}\right)=\frac{1}{m n} \sum_{i=1}^{m} \sum_{j=1}^{n} C E_{i j}\left(\tilde{R}^{h}, \tilde{R}^{l}\right)
$$

For a given expert, $e_{h}$, his/her consensus degree with the group is defined as:

$$
A C D^{h}=\frac{1}{k-1} \sum_{l=1, h \neq l}^{k} C D\left(\tilde{R}^{h}, \tilde{R}^{l}\right)
$$

Notice that it is $A C D^{h}=\frac{1}{m} \sum_{i=1}^{m} A C A_{i}^{h}$. In general, the greater the value of $A C D^{h}$, the higher the agreement between the individual expert $e_{h}$ and the group. If $A C D^{h}=1$, then there is total agreement among the group, which means all experts have the same preference values on all the alternatives for all the attributes considered.

Total agreement is rare to occur in practice, which led 


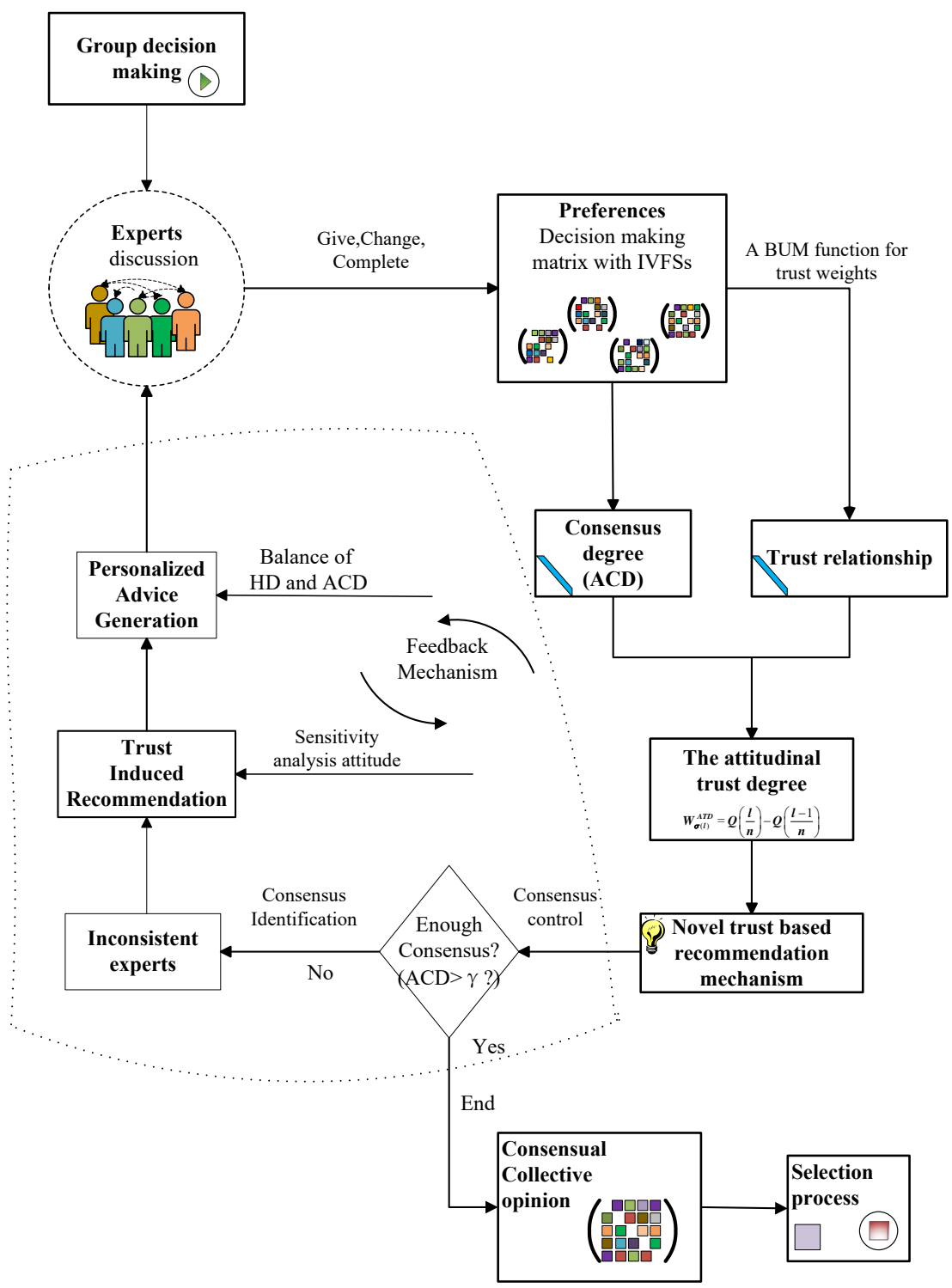

Fig. 1: Attitudinal trust induced recommendation mechanism

Kacprzyk [16] to propose the flexible concept of 'soft consensus'. Consequently, a threshold value $(\gamma \in[0.5,1])$ is set by a group beforehand, so that when the group agreement, as measured above, reaches such threshold value the group is considered to have reached consensus, and the aggregation of the individual decision matrices into a collective one can be carried out.

The following example illustrates how the different three level of consensus defined above are computed in detail. For simplicity, a small number of alternatives and attributes are considered.

Example 1. "A company organizes group tours. There are three scenic spots $A_{i}(i=1,2,3)$ to be selected. Three attributes are available to evaluate the scenic spot: $c_{1}-$ Landscape quality; $c_{2}$-Service quality; $c_{3}$-The price level, with associated weights $w=(0.2,0.5,0.3)^{T}$. Five representatives from different departments form the set of experts $e_{h}(h=1,2,3,4,5)$, and the following interval-valued intu- itionistic fuzzy sets are provided as their preference assessment, respectively:

$$
\begin{aligned}
& \tilde{R}^{(1)}= \\
& \left(\begin{array}{ccc}
([0.3,0.4],[0.4,0.6]) & ([0.5,0.6],[0.1,0.3]) & ([0.3,0.5],[0.2,0.4]) \\
([0.2,0.5],[0.2,0.4]) & ([0.3,0.7],[0.2,0.3]) & ([0.6,0.7],[0.2,0.3]) \\
([0.5,0.7],[0.1,0.3]) & ([0.7,0.8],[0.1,0.2]) & ([0.3,0.4],[0.3,0.5])
\end{array}\right) \\
& \tilde{R}^{(2)}= \\
& \left(\begin{array}{ccc}
([0.2,0.4],[0.3,0.5]) & ([0.3,0.6],[0.2,0.4]) & ([0.4,0.6],[0.2,0.3]) \\
([0.5,0.7],[0.1,0.2]) & ([0.3,0.4],[0.2,0.4]) & ([0.4,0.5],[0.3,0.4]) \\
([0.5,0.6],[0.5,0.4]) & ([0.5,0.6],[0.1,0.3]) & ([0.4,0.5],[0.2,0.3])
\end{array}\right) \\
& \tilde{R}^{(3)}= \\
& \left(\begin{array}{ccc}
([0.6,0.8],[0.1,0.2]) & ([0.2,0.3],[0.5,0.6]) & ([0.1,0.3],[0.5,0.6]) \\
([0.2,0.3],[0.4,0.6]) & ([0.1,0.3],[0.4,0.7]) & ([0.7,0.8],[0.1,0.2]) \\
([0.2,0.4],[0.5,0.6]) & ([0.2,0.3],[0.5,0.7]) & ([0.1,0.2],[0.5,0.7])
\end{array}\right)
\end{aligned}
$$


$\tilde{R}^{(4)}=$

$\left(\begin{array}{lll}([0.5,0.7],[0.2,0.3]) & ([0.1,0.4],[0.4,0.5]) & ([0.2,0.4],[0.3,0.5])\end{array}\right)$ $([0.1,0.4],[0.3,0.5])([0.3,0.4],[0.2,0.5])([0.3,0.5],[0.2,0.4])$ $([0.3,0.5],[0.2,0.4])([0.1,0.3],[0.5,0.6])([0.2,0.3],[0.4,0.6]))$

$\tilde{R}^{(5)}=$

$\left(\begin{array}{lll}([0.3,0.4],[0.4,0.5]) & ([0.4,0.5],[0.3,0.5]) & ([0.2,0.4],[0.3,0.6])\end{array}\right)$ $([0.5,0.7],[0.1,0.3])([0.6,0.8],[0.1,0.2])([0.5,0.6],[0.2,0.3])$

$\left.\begin{array}{lll}([0.5,0.6],[0.2,0.4]) & ([0.4,0.7],[0.2,0.3]) & ([0.3,0.6],[0.3,0.3])\end{array}\right)$

According to expression (5), the average consensus degree on elements are:

$$
\begin{aligned}
& A C E_{i j}^{1}=\left(\begin{array}{lll}
0.835 & 0.788 & 0.869 \\
0.844 & 0.831 & 0.825 \\
0.838 & 0.694 & 0.863
\end{array}\right) \\
& A C E_{i j}^{2}=\left(\begin{array}{lll}
0.831 & 0.850 & 0.813 \\
0.781 & 0.856 & 0.863 \\
0.887 & 0.775 & 0.819
\end{array}\right) \\
& A C E_{i j}^{3}=\left(\begin{array}{lll}
0.725 & 0.794 & 0.813 \\
0.769 & 0.719 & 0.750 \\
0.756 & 0.700 & 0.763
\end{array}\right) \\
& A C E_{i j}^{4}=\left(\begin{array}{lll}
0.800 & 0.831 & 0.894 \\
0.806 & 0.850 & 0.863 \\
0.875 & 0.700 & 0.838
\end{array}\right) \\
& A C E_{i j}^{5}=\left(\begin{array}{lll}
0.844 & 0.863 & 0.888 \\
0.800 & 0.719 & 0.900 \\
0.894 & 0.781 & 0.819
\end{array}\right)
\end{aligned}
$$

Applying expression (7), the average consensus degrees on alternatives are:

$A C A_{i}^{1}=(0.827,0.833,0.798) ; \quad A C A_{i}^{2}=(0.831,0.833,0.827) ;$ $A C A_{i}^{3}=(0.777,0.746,0.740) ; \quad A C A_{i}^{4}=(0.842,0.840,0.804) ;$

$A C A_{i}^{\mathrm{k}}=(0.865,0.806,0.831)$.

Finally, the experts' consensus degrees are:

$$
\begin{gathered}
A C D^{1}=0.819, A C D^{2}=0.831, A C D^{3}=0.754, \\
A C D^{4}=0.829, A C D^{5}=0.834 .
\end{gathered}
$$

If the threshold value of consensus is set at 0.75 , then group is at a state of consensus. However, if $\gamma=0.8$, then group has not reached consensus yet because one expert $\left(e_{3}\right)$ has a consensus degree with the group below such threshold value. Therefore a recommendation mechanism is required to assist such expert to increase his/her consensus degree."

In what follows, a new trust-inducted recommendation mechanism is proposed to produce personalized advice to inconsistent experts to increase their consensus degree.

\section{B. Attitudinal trust induced recommendation mechanism}

The new trust induced recommendation mechanism considers the trust weight of experts generally, and it consists of the following three steps: (1) identification of the inconsistent experts; (2) attitudinal trust model; and (3) generation of personalized advice by attitudinal trust.

1) Identification of the inconsistent expert: The experts with a consensus degree lower than the threshold value $\gamma$ are identified as inconsistent: $E X P C H=\left\{h \mid C D^{h}<\gamma \in\right.$ $[0.5,1]\}$.
Example 2 (Example 1 continuation). "Setting the consensus threshold value $\gamma=0.8$, we have $A C D^{3}<\gamma$, then, the expert $e_{3}$ is inconsistent and need adjustment. Therefore, the recommendation mechanism must be activated to generate advice to assist expert $e_{3}$ to increase his/her consensus degree."

2) Attitudinal trust model: In order to quantify the trust relationship between experts, the definition of trust matrix is constructed by measuring the pairwise similarity of their opinions (modelled here as interval-valued intuitionistic fuzzy numbers).

Definition 4 (Trust matrix). "For experts $e^{h}$ and $e^{l}$, their trust relationship is a measure of their consensus degree, i.e.

$$
T R_{h l}=C D\left(\tilde{R}^{h}, \tilde{R}^{l}\right)
$$

The trust relationship matrix $T R M=\left(T R_{h l}\right)_{k \times k}$ is constructed."

An expert will naturally have different trust relationships with different experts. The closer the opinions of two experts, the higher the trust degree between them will be. As aforementioned, the binary trust model considers only two states of trust: 'trusting' and 'distrusting'. To model the trust relationship generally, an attitudinal trust degree is defined as follows.

Definition 5 (Attitudinal trust degree). Let the trust relationship of expert $e_{h}$ with other experts be $\left\{T R_{h l} \mid l=\right.$ $1,2, \ldots, k, h \neq l\}$. Let $\sigma:\{1,2, \ldots, k-1\} \rightarrow\{l=$ $1,2, \ldots, k \mid h \neq l\}$ be the permutation that verifies $T R_{h \sigma(t)} \geq$ $T R_{h \sigma(t+1)}$. The attitudinal trust degree (ATD) is calculated using an OWA operator guided by a basic unit-monotonic (BUM) function $Q$ to derive the aggregating weighted values

$$
W_{\sigma(t)}^{A T D}=Q\left(\frac{t}{k-1}\right)-Q\left(\frac{t-1}{k-1}\right) ; t=1,2, \ldots, k-1
$$

Notice that if $T R_{h \sigma(m)}$ is selected as the trust threshold value and the Window-OWA (W-OWA) operator [46] is used, i.e. if the following weighting vector $W^{A T D}=$ $(\underbrace{1 / m, \ldots, 1 / m}_{m}, \underbrace{0, \ldots, 0}_{k-1-m})$ is used in Def. 5, then the binary trust model would derive because the experts will be classified into two groups of 'trusting' experts ( $m$ experts with highest trust relationship) and 'distrusting' experts (the rest of experts).

In [47], Yager proposed the following parameterized family of regular increasing monotone (RIM) quantifiers $Q(r)=r^{\alpha}$, with $\alpha \in[0,1]$ being an attitudinal parameter which reflects the attitude of the expert. Chiclana et al. proved in [7] that the concavity property of this type of RIM quantifiers implies:

$$
W_{\sigma(1)}^{A T D} \geq W_{\sigma(2)}^{A T D} \geq \cdots \geq W_{\sigma(k-1)}^{A T D}
$$

Furthermore, when $\alpha \in[0,1]$ we have orness $\left(W^{A T D}\right)=$ $1 /(1+\alpha)$; thus, the lower $\alpha$ is, the closer the OWA aggregation guided by the corresponding $Q$ will be to the maximum aggregation operator. This parameterized family of RIM quantifiers will be used in the rest of the paper. 
Example 3 (Example 2 continuation). "According to definition 4, the following trust relationship matrix is obtained:

$$
T R M=\left(\begin{array}{lllll}
1.000 & 0.883 & 0.700 & 0.808 & 0.886 \\
0.883 & 1.000 & 0.717 & 0.819 & 0.903 \\
0.700 & 0.717 & 1.000 & 0.869 & 0.731 \\
0.808 & 0.819 & 0.869 & 1.000 & 0.817 \\
0.886 & 0.903 & 0.731 & 0.817 & 1.000
\end{array}\right)
$$

Table I shows the different trust weights for the inconsistent expert $e_{3}$ according to different attitudinal parameter $\alpha$ for $Q(r)=r^{\alpha}, \alpha \in[0,1]$ and expression (11).

TABLE I: Trust weights with different $\alpha$ values

\begin{tabular}{l|ccccccc}
\hline \multicolumn{1}{c|}{$\alpha$} & 0 & $1 / 6$ & $2 / 6$ & $3 / 6$ & $4 / 6$ & $5 / 6$ & 1 \\
\hline$W_{\sigma(1)}^{A T D}$ & 1 & 0.79 & 0.63 & 0.5 & 0.4 & 0.31 & 0.25 \\
\hline$W_{\sigma(2)}^{A T D}$ & 0 & 0.1 & 0.16 & 0.21 & 0.23 & 0.25 & 0.25 \\
\hline$W_{\sigma(3)}^{A T D}$ & 0 & 0.06 & 0.12 & 0.16 & 0.2 & 0.23 & 0.25 \\
\hline$W_{\sigma(4)}^{A T D}$ & 0 & 0.05 & 0.09 & 0.13 & 0.17 & 0.21 & 0.25 \\
\hline
\end{tabular}

The attitudinal trust degree (ATD) assigns different trust weight according to the attitudinal parameter $\alpha$. Therefore, the trust degree can be defined generally in a continuous scale. In particular, we have:

(1) if $Q(r)=r^{0}$, then $W^{A T D}=(1,0 \ldots, 0)$, which corresponds to the 'optimistic trust policy' by which an inconsistent expert trusts only the expert with closest opinions to him/her. (2) if let $Q(r)=r$, then $W^{A T D}=\left(\frac{1}{k-1}, \ldots, \frac{1}{k-1}\right)$, which corresponds to the 'indifferent trust policy' by which an inconsistent expert trusts all other experts equally. This case corresponds to the the traditional recommendation mechanism based on the arithmetic mean operator."

The ATD can be regarded a reliable aggregation method of individual decision matrices into a collective one.

Definition 6. Let $\tilde{R}^{l}=\left(\tilde{r}_{i j}^{l}\right)_{m \times n}, l=1, \ldots, k$ be a set of interval-valued fuzzy decision matrices and $\tilde{R}^{h}$ be decision matrix of the inconsistent expert $e_{h}$. Let the trust relationship of expert $e_{h}$ with other experts be $\left\{T R_{h l} \mid l=1,2, \ldots, k, h \neq\right.$ $l\}$ and $\sigma:\{1,2, \ldots, k-1\} \rightarrow\{l=1,2, \ldots, k \mid h \neq l\}$ the permutation that verifies $T R_{h \sigma(t)} \geq T R_{h \sigma(t+1)}$. The matrix $\bar{R}^{c}=\left(\bar{r}_{i j}^{c}\right)_{k \times k}$ with element

$$
\begin{gathered}
\bar{r}_{i j}^{c}=\left(\bar{\mu}_{i j}^{c}, \bar{v}_{i j}^{c}\right) ; \\
\bar{\mu}_{i j}^{c}=\sum_{t=1}^{k-1} W_{\sigma(t)}^{A T D} \tilde{\mu}_{i j}^{\sigma(t)} \wedge \bar{v}_{i j}^{c}=\sum_{t=1}^{k-1} W_{\sigma(t)}^{A T D} \tilde{v}_{i j}^{\sigma(t)}
\end{gathered}
$$

where $\sum_{t=1}^{k-1} W_{\sigma(t)}^{A T D}=1$, is the collective matrix aggregated by $A T D$, and it will be denoted as

$$
\bar{R}^{c}=\sum_{t=1}^{k-1} W_{\sigma(t)}^{A T D} \tilde{R}^{\sigma(t)} .
$$

In what follows, matrix $\bar{R}^{c}$ will be proved to increase the group consensus degree. Before this, the following lemma is recalled [23].

Lemma 1. "For ordered vector $x=\left(x_{1}, x_{2}, \ldots, x_{n}\right), x_{1} \geq$ $x_{2} \geq \ldots \geq x_{n}$, weights $w=\left(w_{1}, w_{2}, \ldots, w_{n}\right)$, and vector $\left(\alpha_{1}, \alpha_{2}, \ldots, \alpha_{n}\right), \alpha_{i} \geq 0 \forall i:$

1) If $w_{1} \geq w_{2} \geq \ldots \geq w_{n}$, then

$$
\begin{aligned}
& \alpha_{1} w_{1} x_{1}+\alpha_{2} w_{2} x_{2}+\ldots+\alpha_{n} w_{n} x_{n} \\
& \quad \geq \frac{\alpha_{1} w_{1}+\ldots+\alpha_{n} w_{n}}{\alpha_{1}+\ldots+\alpha_{n}}\left(\alpha_{1} x_{1}+\ldots+\alpha_{n} x_{n}\right)
\end{aligned}
$$

2) If $w_{1} \leq w_{2} \leq \ldots \leq w_{n}$, then

$$
\begin{aligned}
& \alpha_{1} w_{1} x_{1}+\alpha_{2} w_{2} x_{2}+\ldots+\alpha_{n} w_{n} x_{n} \\
& \quad \leq \frac{\alpha_{1} w_{1}+\ldots+\alpha_{n} w_{n}}{\alpha_{1}+\ldots+\alpha_{n}}\left(\alpha_{1} x_{1}+\ldots+\alpha_{n} x_{n}\right)
\end{aligned}
$$

with equality if and only if $x_{1}=x_{2}=\ldots=x_{n}$."

A desirable property of the consensus level is presented below.

Proposition 1. Let $\tilde{R}^{l}=\left(\tilde{r}_{i j}^{l}\right)_{m \times n}, l=1, \ldots, k$ be a set of interval-valued fuzzy decision matrices, and $\bar{R}^{c}$ the collective matrix aggregated by ATD. Then

$$
C D\left(\tilde{R}^{h}, \bar{R}^{c}\right) \geq \frac{1}{k-1} \sum_{l=1, l \neq h}^{k} C D\left(\tilde{R}^{h}, \tilde{R}^{l}\right)
$$

Proof. From expression (4), it is

$$
C D\left(\tilde{R}^{h}, \bar{R}^{c}\right)=1-\frac{1}{m n} \sum_{i=1}^{m} \sum_{j=1}^{n} d\left(\tilde{r}_{i j}^{h}, \tilde{r}_{i j}^{l}\right),
$$

with $d\left(\tilde{r}_{i j}^{h}, \tilde{r}_{i j}^{l}\right)=\frac{\left|\mu_{i j}^{h-}-\mu_{i j}^{l-}\right|+\left|\mu_{i j}^{h+}-\mu_{i j}^{l+}\right|+\left|v_{i j}^{h-}-v_{i j}^{l-}\right|+\left|v_{i j}^{h+}-v_{i j}^{l+}\right|}{4}$.

From Def. 6, it is $\bar{r}_{i j}^{c}=\left(\bar{\mu}_{i j}^{c}, \bar{v}_{i j}^{c}\right) ; \bar{\mu}_{i j}^{c}=\sum_{t=1}^{k-1} W_{\sigma(t)}^{A T D} \tilde{\mu}_{i j}^{\sigma(t)}$ and $\bar{v}_{i j}^{c}=\sum_{t=1}^{k-1} W_{\sigma(t)}^{A T D} \tilde{v}_{i j}^{\sigma(t)}$, where $\sigma:\{1,2, \ldots, k-1\} \rightarrow$ $\{l=1,2, \ldots, k \mid h \neq l\}$ is the permutation that verifies $T R_{h \sigma(t)} \geq T R_{h \sigma(t+1)}$, i.e. $C D\left(\tilde{R}^{h}, \tilde{R}^{\sigma(t)}\right) \geq$ $C D\left(\tilde{R}^{h}, \tilde{R}^{\sigma(t+1)}\right)$. Applying the triangle inequality, we have:

$$
\begin{aligned}
\left|\mu_{i j}^{h-}-\bar{\mu}_{i j}^{c-}\right| & =\left|\sum_{t=1}^{k-1} W_{\sigma(t)}^{A T D}\left(\mu_{i j}^{h-}-\mu_{i j}^{\sigma(t)-}\right)\right| \\
& \leq \sum_{t=1}^{k-1} W_{\sigma(t)}^{A T D}\left|\mu_{i j}^{h-}-\mu_{i j}^{\sigma(t)-}\right| .
\end{aligned}
$$

Similar expressions are obtained for $\left|\mu_{i j}^{h+}-\bar{\mu}_{i j}^{c+}\right|$, $\left|v_{i j}^{h-}-\bar{v}_{i j}^{c-}\right|$ and $\left|v_{i j}^{h+}-\bar{v}_{i j}^{c+}\right|$. Thus, it is

$$
\begin{aligned}
& C D\left(\tilde{R}^{h}, \bar{R}^{c}\right) \geq 1-\frac{1}{m n} \sum_{i=1}^{m} \sum_{j=1}^{n} \sum_{t=1}^{k-1} W_{\sigma(t)}^{A T D} \times \\
& \\
& \frac{\left|\mu_{i j}^{h-}-\mu_{i j}^{\sigma(l)-}\right|+\left|\mu_{i j}^{h+}-\mu_{i j}^{\sigma(l)+}\right|+\left|v_{i j}^{h-}-v_{i j}^{\sigma(l)-}\right|+\left|v_{i j}^{h+}-v_{i j}^{\sigma(l)+}\right|}{4}
\end{aligned}
$$

The right hand side of the above inequality can be re-written as $\sum_{t=1}^{k-1} W_{\sigma(t)}^{A T D} C D\left(\tilde{R}^{h}, \bar{R}^{\sigma(t)}\right)$. Therefore, it is:

$$
C D\left(\tilde{R}^{h}, \bar{R}^{c}\right) \geq \sum_{t=1}^{k-1} W_{\sigma(t)}^{A T D} C D\left(\tilde{R}^{h}, \bar{R}^{\sigma(t)}\right)
$$

Because $W_{\sigma(t)}^{A T D} \geq W_{\sigma(t+1)}^{A T D}$ and $C D\left(\tilde{R}^{h}, \tilde{R}^{\sigma(t)}\right) \geq$ $C D\left(\tilde{R}^{h}, \tilde{R}^{\sigma(t+1)}\right)$, Lemma 1 Case 1 with vector $\mathbf{1}_{1 \times(k-1)}$ 
yields:

$$
\begin{aligned}
\sum_{t=1}^{k-1} W_{\sigma(t)}^{A T D} C D\left(\tilde{R}^{h}, \bar{R}^{\sigma(t)}\right) \\
\geq \frac{\sum_{t=1}^{k-1} W_{\sigma(t)}^{A T D}}{k-1} \sum_{t=1}^{k-1} C D\left(\tilde{R}^{h}, \bar{R}^{\sigma(t)}\right)
\end{aligned}
$$

Finally, because $\sum_{t=1}^{k-1} W_{\sigma(t)}^{A T D}=1$, we conclude that

$$
\sum_{t=1}^{k-1} W_{\sigma(t)}^{A T D} C D\left(\tilde{R}^{h}, \bar{R}^{\sigma(t)}\right) \geq \frac{1}{k-1} \sum_{t=1}^{k-1} C D\left(\tilde{R}^{h}, \bar{R}^{\sigma(t)}\right)
$$

Consequently, it is:

$$
C D\left(\tilde{R}^{h}, \bar{R}^{c}\right) \geq \frac{1}{k-1} \sum_{l=1, l \neq h}^{k} C D\left(\tilde{R}^{h}, \tilde{R}^{l}\right)
$$

When $Q(r)=r$, we have $W^{A T D}=\left(\frac{1}{k-1}, \ldots, \frac{1}{k-1}\right)$ and $\bar{R}^{c}$ becomes the average interval-valued fuzzy decision matrix $\bar{R}$. Because the average is bounded below by the minimum, the following corollary is derived.

Corollary 1. Let $\tilde{R}^{l}=\left(\tilde{r}_{i j}^{l}\right)_{m \times n}, l=1, \ldots, z$ be intervalvalued intuitionistic fuzzy decision matrices, and $\bar{R}$ be their average interval-valued fuzzy decision matrix. Then

$$
C D\left(\tilde{R}^{h}, \bar{R}\right) \geq \min _{k}\left\{C D\left(\tilde{R}^{h}, \tilde{R}^{l}\right)\right\} .
$$

3) Generation of personalized advice by attitudinal trust: The feedback mechanism proposed in this paper adjusts the preference values of the inconsistency experts so as to accelerate the convergence of the consensus process. For all $h \in E X P C H$, personalized advice generated for expert $e_{h}$ are:

"In order to increase your consensus level $(C D)$, you are advised to change your preference value $r_{i j}^{h}=\left(\tilde{\mu}_{i j}^{h}, \tilde{v}_{i j}^{h}\right)$ to

$$
\left(r \tilde{\mu}_{i j}^{h}, r \tilde{v}_{i j}^{h}\right)=\left((1-\delta) \tilde{\mu}_{i j}^{h}+\delta \bar{\mu}_{i j}^{c},(1-\delta) \tilde{v}_{i j}^{h}+\delta \bar{v}_{i j}^{c}\right)
$$

where $\bar{\mu}_{i j}^{c}=\sum_{t=1}^{k-1} W_{\sigma(t)}^{A T D} \tilde{\mu}_{i j}^{\sigma(t)}$ and $\bar{v}_{i j}^{c}=\sum_{t=1}^{k-1} W_{\sigma(l)}^{A T D} \tilde{v}_{i j}^{\sigma(t)}$ and $\delta \in[0,1]$ is the feedback parameter that controls the level of change from the original opinion into the collective aggregated opinion by ATD."

The following results shows that adopting the attitudinal trust recommendation mechanism guarantees the increase of the consensus degree of the most inconsistent expert.

Proposition 2. Let $\tilde{R}^{1}, \tilde{R}^{2}, \tilde{R}^{3}, \ldots, \tilde{R}^{k}$ be the expert's original evaluation matrices. If the most inconsistent expert $e_{h}$ $\left(A C D^{h} \leq A C D^{l}, l \neq h ; l=1, \ldots, k\right)$ adopts the ATD based $R \tilde{R}^{h}$ as his/her new decision matrix and, then

$$
A C D^{h} \leq \overline{A C D}^{h}
$$

where $\overline{A C D}^{h}$ is the new ATD based consensus degree of expert $e_{h}$.
Proof. According to expression (16), we have

$$
R \tilde{R}^{h}=(1-\delta) \tilde{R}^{h}+\delta \sum_{t=1}^{k-1} W_{\sigma(t)}^{A T D} \tilde{R}^{\sigma(t)}
$$

where $\sigma:\{1,2, \ldots, k-1\} \rightarrow\{l=1,2, \ldots, k \mid h \neq l\}$ be the permutation that verifies $T R_{h \sigma(t)} \geq T R_{h \sigma(t+1)}$. Recall from (9) and (8) that

$$
\begin{aligned}
A C D^{h} & =\frac{1}{k-1} \sum_{l=1, h \neq l}^{k} C D\left(\tilde{R}^{h}, \tilde{R}^{l}\right) \\
& =\frac{1}{k-1} \sum_{t=1}^{k-1}\left(1-\left|\tilde{R}^{h}-\tilde{R}^{\sigma(t)}\right|\right) \\
& =1-\frac{1}{k-1} \sum_{t=1}^{k-1}\left|\tilde{R}^{h}-\tilde{R}^{\sigma(t)}\right| .
\end{aligned}
$$

Accordingly,

$$
\overline{A C D}^{h}=1-\frac{1}{k-1} \sum_{t=1}^{k-1}\left|R \tilde{R}^{h}-\tilde{R}^{\sigma(t)}\right| .
$$

Applying the triangle inequality, it is

$$
\begin{aligned}
& \left|R \tilde{R}^{h}-\tilde{R}^{\sigma(t)}\right|=\left|(1-\delta) \tilde{R}^{h}+\delta \sum_{s=1}^{k-1} W_{\sigma(s)}^{A T D} \tilde{R}^{\sigma(s)}-\tilde{R}^{\sigma(t)}\right| \\
& =\left|(1-\delta)\left(\tilde{R}^{h}-\tilde{R}^{\sigma(t)}\right)+\delta\left(\sum_{s=1}^{k-1} W_{\sigma(s)}^{A T D}\left(\tilde{R}^{\sigma(s)}-\tilde{R}^{\sigma(t)}\right)\right)\right| \\
& \leq(1-\delta)\left|\tilde{R}^{h}-\tilde{R}^{\sigma(t)}\right|+\delta\left|\sum_{s=1}^{k-1} W_{\sigma(s)}^{A T D}\left(\tilde{R}^{\sigma(s)}-\tilde{R}^{\sigma(t)}\right)\right| \\
& \leq(1-\delta)\left|\tilde{R}^{h}-\tilde{R}^{\sigma(t)}\right|+\delta \sum_{s=1}^{k-1} W_{\sigma(s)}^{A T D}\left|\tilde{R}^{\sigma(s)}-\tilde{R}^{\sigma(t)}\right| .
\end{aligned}
$$

Thus:

$$
\begin{aligned}
& \sum_{t=1}^{k-1}\left|R \tilde{R}^{h}-\tilde{R}^{\sigma(t)}\right| \\
& \leq(1-\delta) \sum_{t=1}^{k-1}\left|\tilde{R}^{h}-\tilde{R}^{\sigma(t)}\right|+\delta \sum_{t=1}^{k-1} \sum_{s=1}^{k-1} W_{\sigma(s)}^{A T D}\left|\tilde{R}^{\sigma(s)}-\tilde{R}^{\sigma(t)}\right| \\
& =(1-\delta) \sum_{t=1}^{k-1}\left|\tilde{R}^{h}-\tilde{R}^{\sigma(t)}\right|+\delta \sum_{s=1}^{k-1} W_{\sigma(s)}^{A T D}\left(\sum_{t=1}^{k-1}\left|\tilde{R}^{\sigma(s)}-\tilde{R}^{\sigma(t)}\right|\right) .
\end{aligned}
$$

Because $A C D^{h} \leq A C D^{l}, \forall l \neq h ;(l=1, \ldots, k)$, then it is

$$
\sum_{t=1}^{k-1}\left|\tilde{R}^{\sigma(t)}-\tilde{R}^{h}\right| \geq \sum_{t=1}^{k-1}\left|\tilde{R}^{\sigma(t)}-\tilde{R}^{\sigma(s)}\right| ; \forall s .
$$

Using the following equality

$$
\sum_{t=1}^{k-1}\left|\tilde{R}^{\sigma(t)}-\tilde{R}^{h}\right|=\sum_{s=1}^{k-1} W_{\sigma(s)}^{A T D}\left(\sum_{t=1}^{k-1}\left|\tilde{R}^{\sigma(t)}-\tilde{R}^{h}\right|\right),
$$

it is

$$
\sum_{t=1}^{k-1}\left|\tilde{R}^{\sigma(t)}-\tilde{R}^{h}\right| \geq \sum_{s=1}^{k-1} W_{\sigma(s)}^{A T D}\left(\sum_{t=1}^{k-1}\left|\tilde{R}^{\sigma(s)}-\tilde{R}^{\sigma(t)}\right|\right) .
$$

We conclude that

$$
\begin{aligned}
& \sum_{t=1}^{k-1}\left|R \tilde{R}^{h}-\tilde{R}^{\sigma(t)}\right| \leq(1-\delta) \sum_{t=1}^{k-1}\left|\tilde{R}^{h}-\tilde{R}^{\sigma(t)}\right| \\
& +\delta \sum_{t=1}^{k-1}\left|\tilde{R}^{h}-\tilde{R}^{\sigma(t)}\right|=\sum_{t=1}^{k-1}\left|\tilde{R}^{h}-\tilde{R}^{\sigma(t)}\right| .
\end{aligned}
$$


Therefore it is true that

$$
\overline{A C D}^{h} \geq A C D^{h} .
$$

Example 4 ((Example 3 continuation)). "For $\delta=0.25$ and attitudinal parameter $\alpha=2 / 6$, the adjusted decision making matrix of expert $e_{3}$ would be:

$R \tilde{R}_{(\alpha=2 / 6)}^{(3)}=$

$\left(\begin{array}{l}([0.55,0.75],[0.14,0.25])([0.20,0.34],[0.46,0.57])([0.13,0.33],[0.44,0.57]) \\ ([0.21,0.35,[0.36,0.56)\end{array}\right)$ $([0.21,0.35],[0.36,0.56])([0.16,0.35],[0.35,0.63])([0.62,0.73],[0.13,0.24]))$

This yield a value of $A \overline{C D}_{\alpha=2 / 6}^{3}=0.803>\gamma$, and therefore the feedback mechanism is terminated."

4) Sensitivity analysis of consensus with attitudinal parameter: As aforementioned, experts may have different attitude to different experts in the group, i.e. experts might be willing to assign different trust weights to different experts according to the attitudinal parameter $\alpha$, which translate into different consensus degrees.

In particular, we first define the following three representative trust polices:

(1) 'extreme trust policy' by which an inconsistent expert trusts only the expert with closest opinions to his/her opinions;

(2) 'bounded trust policy' by which an inconsistent expert selects the parameter $\alpha$ value that produces a consensus degree equal to the threshold value;

(3) 'indifferent trust policy' by which an inconsistent expert trusts all other experts equally.

Then, three graphical simulations of the future consensus, following the implementation of the recommended values, are provided in Fig. 2.

(1) In Fig. 2a, $\alpha=0$ and then expert $e_{3}$ adopts the 'extreme trust policy' and $e_{3}$ achieves the minimum consensus degree $\overline{A C D}_{0}^{3}=0.790$.

(2) The $\alpha$ value that produces a consensus degree equal to the threshold value is referred to as the value of the 'bounded trust policy'. In our case, the value $\alpha=1 / 6$ returns the consensus degree $\overline{A C D}_{1 / 6}^{3}=0.8$, which is equal to the threshold value $\gamma$ and therefore is the value that corresponds to the 'bounded trust policy', which is shown in Fig. $2 b$.

(3) In Fig. 2c, $\alpha=1$ and then the consistent expert $e_{3}$ adopts the 'indifferent trust policy' as all other experts receive the same trust weight from expert $e_{3}$, whom achieves the maximum consensus degree $\overline{A C D}_{1}^{3}=0.815$.

And then Table II below shows different consensus degrees for expert $e_{3}$ according to different values of the parameter $\alpha$ once the feedback recommended preference values are implemented. Notice that the consensus degree of the (now) consistent expert $e_{3}$ after adopting the feedback recommendations increases with the attitudinal parameter $\alpha$.

\section{RATIONALITY ANALYSIS OF THE ATTITUDINAL TRUST RECOMMENDATION MECHANISM}

This section first analyzes the relationship between our proposed attitudinal trust recommendation mechanism and
TABLE II: Consensus degree with different attitudinal parameter $\alpha$

\begin{tabular}{c|ccccccc}
\hline$\alpha$ & 0 & $1 / 6$ & $2 / 6$ & $3 / 6$ & $4 / 6$ & $5 / 6$ & 1 \\
\hline$\overline{A C D}_{\alpha}^{3}$ & 0.790 & 0.80 & 0.803 & 0.807 & 0.810 & 0.812 & 0.815 \\
\hline
\end{tabular}

the traditional feedback mechanism. Then the definition of harmony degree (HD) is introduced to measure the degree of deviation before and after the change of opinion. Using $\mathrm{HD}$, a comparison between the adjustment cost of attitudinal trust recommendation mechanism and the traditional feedback mechanism is carried out.

\section{A. Traditional recommendation mechanism}

When $\alpha=1$ (see Table I) the RIM quantifier produces equal trust weights of all expert and the ATD-OWA becomes the arithmetic average. This proves that the proposed attitudinal trust recommendation mechanism is more general than the traditional feedback mechanism, which is a particular case. In this case, the corresponding new consensus degrees after the traditional feedback mechanism are:

$$
\begin{gathered}
\overline{A C D}_{\alpha=1}^{1}=0.841 ; \overline{A C D}_{\alpha=1}^{2}=0.845 ; \overline{A C D}_{\alpha=1}^{3}=0.815 ; \\
\overline{A C D}_{\alpha=1}^{4}=0.840 ; \overline{A C D}_{\alpha=1}^{5}=0.848 .
\end{gathered}
$$

Clearly, all the experts achieve the consensus threshold value, and as discussed above it corresponds to the 'indifferent trust policy', which in turn helped the inconsistent expert to achieve maximum consensus level after the feedback process (for $\delta=0.25)$. Thus, the traditional recommendation mechanism aims at maximum consensus degree. However, the higher the consensus degree, the bigger the adjustment cost the inconsistent expert affords. However, in realistic cases, apart from group consensus, the inconsistent experts are inclined to modify their original opinions as little as possible, i.e. they aim at minimising their adjustment cost. How to compromise between group consensus position and individual position is the focus of the next section.

\section{B. Group consensus and harmony degree analysis}

The concept of harmony degree (HD) is introduced below. This concept is based on the extent up to to which an expert's original information is maintained after the feedback mechanism is implemented.

Definition 7 (Harmony degree (HD)). "The harmony degree (HD) of expert $e_{h}$ is:

$$
H D^{h}=1-\frac{1}{m n} \sum_{j=1}^{n} \sum_{i=1}^{m} d\left(\tilde{r}_{i j}^{h}, r \tilde{r}_{i j}^{h}\right)
$$

where $\tilde{R}^{h}=\left(\tilde{r}_{i j}^{h}\right)$ and $R \tilde{R}^{h}=\left(r \tilde{r}_{i j}^{h}\right)$ are the decision matrices before and after the GDM feedback mechanism is carried out."

It is $0 \leq H D^{h} \leq 1$. The larger the value of $H D^{h}$, the smaller the deviation between the original opinions of expert $e_{h}$ and the new opinions after adopting the feedback recommendations [41]. 


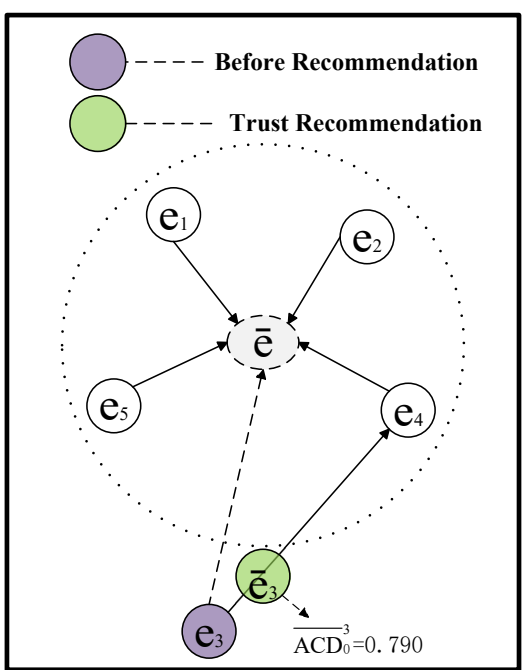

(a) ACD by 'extreme trust policy'

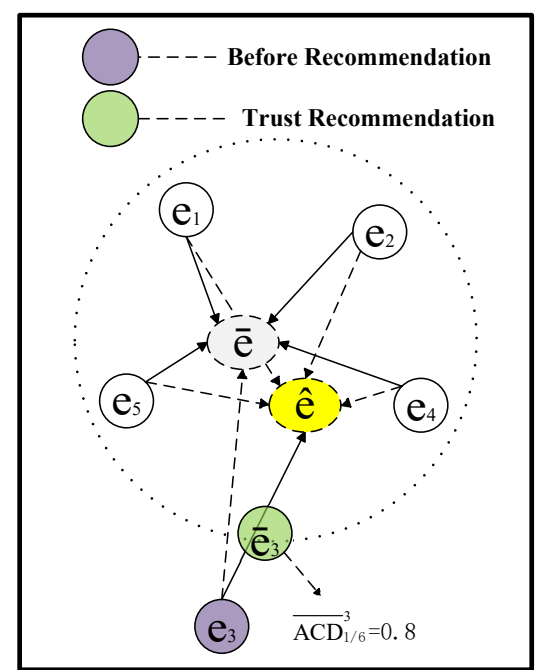

(b) ACD by 'bounded trust policy'

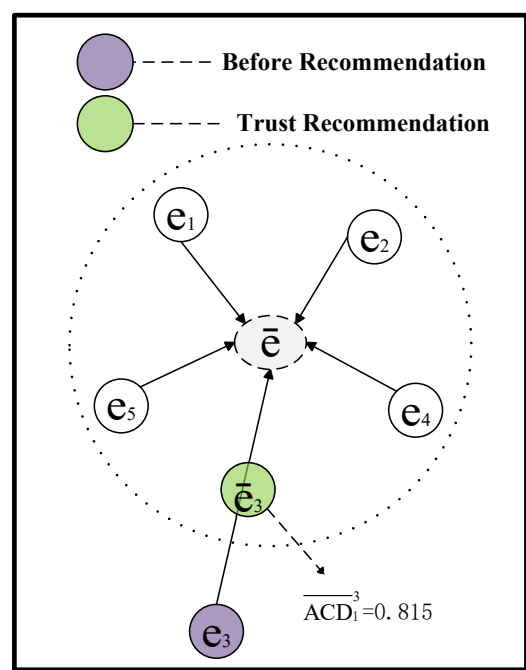

(c) ACD by 'indifferent trust policy'

Fig. 2: Visual simulation of consensus with three trust polices

Proposition 3. Let $\tilde{R}^{1}, \tilde{R}^{2}, \tilde{R}^{3}, \ldots, \tilde{R}^{k}$ be the expert's original evaluation matrices. Assume that expert $e_{h}$ adjusts the original opinion with a new decision matrix $R \tilde{R}^{h}$. Then

$$
H D^{h}>A C D^{h}
$$

Proof. According to expression (16), we have

$$
R \tilde{R}^{h}=(1-\delta) \tilde{R}^{h}+\delta \sum_{t=1}^{k-1} W_{\sigma(t)}^{A T D} \tilde{R}^{\sigma(t)}
$$

where $\sigma:\{1,2, \ldots, k-1\} \rightarrow\{l=1,2, \ldots, k \mid h \neq l\}$ be the permutation that verifies $T R_{h \sigma(t)} \geq T R_{h \sigma(t+1)}$, and therefore $\left|\tilde{R}^{h}-\tilde{R}^{\sigma(t)}\right| \leq\left|\tilde{R}^{h}-\tilde{R}^{\sigma(t+1)}\right|$. It is:

$$
\begin{aligned}
\left|\tilde{R}^{h}-R \tilde{R}^{h}\right| & =\delta\left|\tilde{R}^{h}-\sum_{t=1}^{k-1} W_{\sigma(t)}^{A T D} \tilde{R}^{\sigma(t)}\right| \\
& =\delta\left|\sum_{t=1}^{k-1} W_{\sigma(t)}^{A T D}\left(\tilde{R}^{h}-\tilde{R}^{\sigma(t)}\right)\right| \\
& \leq \delta \sum_{t=1}^{k-1} W_{\sigma(t)}^{A T D}\left|\tilde{R}^{h}-\tilde{R}^{\sigma(t)}\right| .
\end{aligned}
$$

Lemma 1 Case 2 with vector $\mathbf{1}_{1 \times(k-1)}$ yields:

$$
\begin{aligned}
& \sum_{t=1}^{k-1} W_{\sigma(t)}^{A T D}\left|\tilde{R}^{h}-\tilde{R}^{\sigma(t)}\right| \\
& \quad \leq \frac{\sum_{t=1}^{k-1} W_{\sigma(t)}^{A T D}}{k-1}\left(\sum_{t=1}^{k-1}\left|\tilde{R}^{h}-\tilde{R}^{\sigma(t)}\right|\right) \\
& \quad=\frac{1}{k-1}\left(\sum_{t=1}^{k-1}\left|\tilde{R}^{h}-\tilde{R}^{\sigma(t)}\right|\right) .
\end{aligned}
$$

Recall that

$$
A C D^{h}=1-\frac{1}{k-1} \sum_{t=1}^{k-1}\left|\tilde{R}^{h}-\tilde{R}^{\sigma(t)}\right| .
$$

By (16), it is

$$
\begin{aligned}
H D^{h} & =1-\left|\tilde{R}^{h}-R \tilde{R}^{h}\right| \geq 1-\delta\left(1-A C D^{h}\right) \\
& \geq 1-\left(1-A C D^{h}\right)=A C D^{h} .
\end{aligned}
$$

C. Sensitivity analysis of harmony with attitudinal parameter

Contrary to the ACD (see Table II), the HD of an expert decreases with respect to the the attitudinal parameter $\alpha$. In particular, as with Fig. 2, Fig. 3 shows the graphical simulation of the future HD after adopting the recommended values corresponding to the three different trust policies.

(1) In Fig. 3a, if expert $e_{3}$ adopts the 'extreme trust policy', then the maximum HD is achieved: $H D_{0}^{3}=0.967$.

(2) In Fig. 3b, if expert $e_{3}$ adopts the 'bounded trust policy', then she/ he gets an $H D_{1 / 6}^{3}=0.960$.

(3) In Fig. 3c, if expert $e_{3}$ adopts the 'indifferent trust policy', then she/ he gets the minimum HD: $H D_{1}^{3}=0.941$.

Table III below shows the HD of expert $e_{3}$ with different parameter $\alpha$ values. Contrary to the ACD (see Table II), the $\mathrm{HD}$ of an expert decreases with respect to the the attitudinal parameter $\alpha$.

TABLE III: HD of expert $e_{3}$ with different attitudinal parameter $\alpha$

\begin{tabular}{c|ccccccc}
\hline$\alpha$ & 0 & $1 / 6$ & $2 / 6$ & $3 / 6$ & $4 / 6$ & $5 / 6$ & 1 \\
\hline$H D_{\alpha}^{3}$ & 0.967 & 0.960 & 0.957 & 0.952 & 0.947 & 0.944 & 0.941 \\
\hline
\end{tabular}

Using both the $A C D$ and $H D$ simulation analysis, inconsistent experts may adopt the recommendation advices according to their willing, i.e. they can select an appropriate attitudinal parameter to achieve a balance between consensus degree and harmony degree that pleases them. 


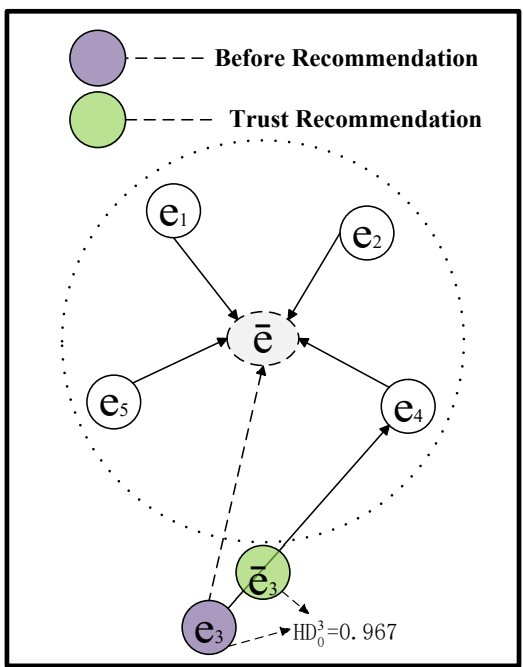

(a) HD by 'extreme trust policy'

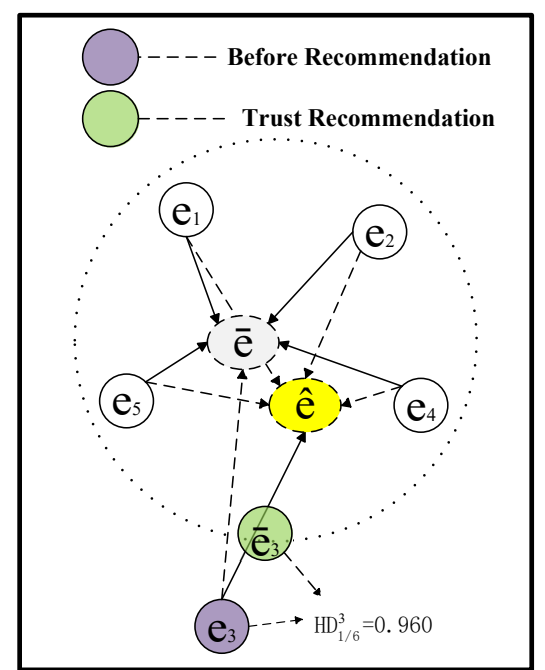

(b) HD by 'bounded trust policy'

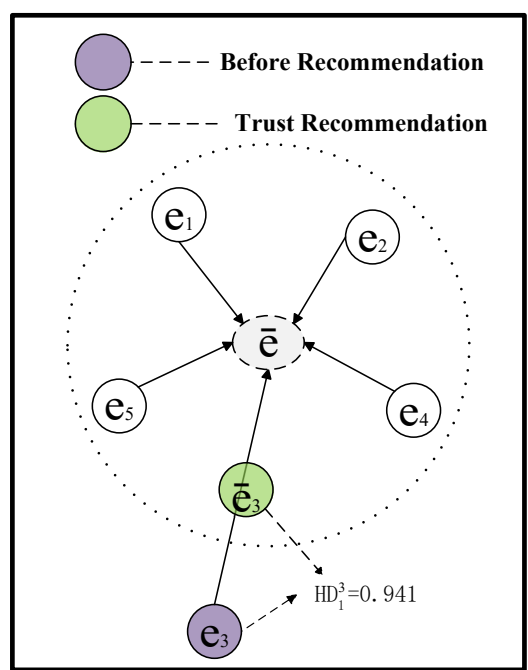

(c) HD by 'indifferent trust policy'

Fig. 3: Visual simulation of HD for three different trust polices

\section{SELECTION PROCESS}

When the consensus degrees of all expert reach the threshold value $\gamma$, then the selection process is implemented. The individual decision matrices $\left\{\tilde{R}^{h} \mid h=1, \ldots, k\right\}$ are aggregated into the group decision matrix $\bar{R}$ from which the final ranking of alternatives is derived.

Example 5 (Example 4 continuation). "Using the attitudinal value $\alpha=2 / 6$, the following collective decision matrix is obtained:

\section{$\bar{R}_{(\alpha=2 / 6)}=$}

$([0.30,0.53],[0.29,0.43])([0.30,0.49],[0.29,0.60])([0.25,0.45],[0.29,0.47])$ $\begin{array}{lll}([0.30,0.53],[0.21,0.39]) & ([0.33,0.53],[0.21,0.41]) & ([0.48,0.61],[0.21,0.33]) \\ ([0.41,0.57],[0.25,0.41]) & ([0.38,0.55],[0.27,0.41]) & ([0.27,0.41],[0.31,0.47])\end{array}$

Then, each of the alternatives is associated the following final attribute weighted assessment

$$
\tilde{r}_{i}=\sum_{j=1}^{n} \omega_{j} \bar{r}_{i j}
$$

Using the criteria weighting vector $\omega=(0.2,0.5,0.3)^{T}$, we derive the following collective overall opinions values $\tilde{r}_{i}(i=$ $1,2,3)$ of the alternatives:

$$
\begin{aligned}
& \tilde{r}_{1}=([0.298,0.484],[0.290,0.455]) ; \\
& \tilde{r}_{2}=([0.372,0.553],[0.209,0.380]) ; \\
& \tilde{r}_{3}=([0.353,0.510],[0.279,0.428]) .
\end{aligned}
$$

The associated IVIFTs scores are: $\widetilde{S}_{X C}\left(\tilde{r}_{1}\right)=$ $0.02 ; \widetilde{S}_{X C}\left(\tilde{r}_{2}\right)=0.17 ; \widetilde{S}_{X C}\left(\tilde{r}_{3}\right)=0.08$. Accordingly, the best alternative can be chosen as $A_{2}$."

\section{THE PROCESS OF CONSENSUS BASED ON ATTITUDINAL TRUST INDUCTION RECOMMENDATION MECHANISM}

In this article, the the attitude trust induction recommendation mechanism for consensus reaching process can be divided into the following six steps, as depicted in Fig. 1.
Step 1. Experts in group decision making respectively express their original evaluation of alternatives by using intervalvalued intuitionistic fuzzy sets in Definition 1 , and then construct each individual preference evaluation matrix.

Step 2. Consensus measure is carried out through equations (4)-(9), and the inconsistent experts are identified by comparing with the threshold value of consensus. Then, a recommendation mechanism is activated to assist such expert to increase his/her consensus degree.

Step 3. According to equations (10)-(12), the attitude trust consensus model of inconsistent experts is established, and the trust weight of inconsistent experts under different parameters is calculated by the equation (11).

Step 4. Then, the feedback mechanism is activated to generate personalized suggestions for inconsistent experts by using equation (16).

Step 5. By ACD index in equation (9) and HD index in equation (17), the sensitivity analysis of consensus degree and harmony degree with attitudinal parameter is explored. Then, a new round of consensus measurement is carried out. If the inconsistent experts can not arrive the threshold value of consensus, then go to step 2, otherwise go to the next step.

Step 6. The individual decision making matrices are aggregated into a collective one, and then the interval-valued intuitionistic fuzzy value of the alternatives is calculated by equation (19). And then, the best alternative is selected by Definition (3).

\section{CONClusion}

This article proposes a trust induced recommendation mechanism by considering the attitude of the inconsistent expert. It has the following main advantages over other consensus model presented in the literature:

1) It presents the definition of attitudinal trust model. Trust weights are assigned to others by taking into account 
the decision attitude, which allows a continuous trust status modelling from ' $h i g h$ ' to 'low' and consequently it is a more general trust information modelling approach than the simple trust model based on the a binary trust relationship: 'trusting' and 'distrusting'. This simple trust model can be obtained as a special case of the proposed attitudinal trust model by generating via the implementation of a window-OWA (W-OWA) operator.

2) It investigates the attitudinal trust induction recommendation mechanism for consensus in GDM. It generates the recommendation advices via the the trust policy: the closer the experts' opinions, the higher the trust degree between them. In particular, three typical policies are proposed to model the trust behavior of experts in the consensus reaching process: 'extreme trust policy', 'bounded trust policy' and 'indifferent trust policy'. The implementation of the attitudinal trust induced recommendations increases the consensus degrees and therefore guarantees that the group opinion converge to consensus.

3) The concept of harmony degree (HD) is introduced to quantify the cost of change of the original opinion after the implementation of the attitudinal trust induced recommendations. The HD index is proved to be bigger than the ACD index, and they behave in a reverse way with respect to the attitudinal parameter. When the attitudinal parameter increase in its domain $[0,1]$, the HD index decreases while the ACD index increases. Combining both the HD index and ACD index, a sensitivity analysis with respect to the attitudinal parameter can be carried out for the inconsistent experts to be able to select an appropriate attitudinal parameter to achieve an acceptable compromise between group consensus degree and their own harmony degree.

Summarising, the attitudinal trust induction feedback mechanism allows to discriminate the trusted experts with attitude and the inconsistent experts to revisit their evaluations according to the advice from trusted experts. Therefore, it is more reasonable than the traditional feedback mechanism which does not consider graduation of trust. However, our trust model dose not take into account the trust relationship from social network. A more reasonable method is to combine the calculated trust model and the social network trust model into a comprehensive one, and then a comprehensive trust based feedback mechanism may be explored in our future work.

\section{ACKNOWLEDGEMENTS}

The authors are very grateful to the anonymous referees for their valuable comments and suggestions to improve considerably the quality of this paper. This work was sponsored by National Natural Science Foundation of China (NSFC) (No. 71571166, 71331002) and Natural Science Foundation of Shanghai (18ZR1416900).

\section{REFERENCES}

[1] Alonso, S., Herrera-Viedma, E., Chiclana, F. and Herrera, F. (2010). A web based consensus support system for group decision making problems and incomplete preferences. Information Sciences 180(23), $4477-4495$.
[2] Alonso, S, Prez, I.J., Cabrerizo, F.J. and Herrera-Viedma, E. (2013). A Linguistic Consensus Model for Web 2.0 Communities. Applied Soft Computing 13 (1), 149-157.

[3] Atanassov, K. and Gargov, G. (1989). Interval-valued intuitionistic fuzzy sets. Fuzzy Sets and Systems 31, 343-349.

[4] Atanassov, K. (1986). Intuitionstic fuzzy sets. Fuzzy Sets and Systems 20, 87-96.

[5] Capuano, N., Chiclana, F., Fujita, H., Herrera-Viedma, E. and Loia, V. (2018). Fuzzy Group Decision Making With Incomplete Information Guided by Social Influence. IEEE Transactions on Fuzzy Systems 26(3), 1704-1718.

[6] Chen, X., Zhang, H. J. and Dong, Y. C. (2015). The fusion process with heterogeneous preference structures in group decision making: A survey. Information Fusion 24, 72-83.

[7] Chiclana, F., Herrera-Viedma, E., Herrera, F., Alonso, S. (2017). Some induced ordered weighted averaging operators and their use for solving group decision-making problems based on fuzzy preference relations. European Journal of Operational Research 182(1), 383-399.

[8] Chu, J. F., Liu, X. W., Wang, Y. M. and Chin, K. S. (2016). A group decision making model considering both the additive consistency and group consensus of intuitionistic fuzzy preference relations. Computers Industrial Engineering 101, 227-242.

[9] Deschrijver, G. and Kerre, E. E. (2003). On the relationship between some extensions of fuzzy set theory, Fuzzy Sets and Systems, 133 227235.

[10] Dong, Y. C., Fan, Z. P. and Yu, S. (2015). Consensus building in a local context for the AHP-GDM with the individual numerical scale and prioritization method. IEEE Transactions on Fuzzy Systems 23(2), 354-368.

[11] Dong, Y. C., Zhang, H. J. and Herrera-Viedma, E. (2016). Consensus reaching model in the complex and dynamic MAGDM problem. Knowledge-based Systems 106, 206-219.

[12] Gong, Z. W., Xu, X. X., Lu, F. L., Li, L. S. and Xu, C. (2015). On consensus models with utility preferences and limited budget. Applied Soft Computing 35, 840-849.

[13] Gong, Z. W., Zhang, H. H., Forrest, J., Li, L. S. and Xu, X. X. (2015). Two consensus models based on the minimum cost and maximum return regarding either all individuals or one individual. European Journal of Operational Research 240, 183-192.

[14] Herrera-Viedma, E., Cabrerizo, F. J., Kacprzyk, J., Pedrycz, W. (2014). A review of soft consensus models in a fuzzy environment. Information Fusion 17, 4-13.

[15] Jin, L. S., Mesiar, R. and Qian, G.(2018). Weighting models togenerate weights and capacities in multicriteria group decision making. IEEE Transactions on Fuzzy Systems 26(4), 2225-2236.

[16] Kacprzyk, J. (1986). Group decision making with a fuzzy linguistic majority. Fuzzy Sets and Systems 18(2), 105-118.

[17] Li, D. F. (2012) Representation theorem and extension principles for Atanassov's intuitionistic fuzzy sets and algebraic operations, Advances in Fuzzy Sets and Systems, 2 255-265.

[18] Li, D. F. (2014) Decision and Game Theory in Management With Intuitionistic Fuzzy Sets, Spring.

[19] Liao, H. C., Xu, Z. S., Zeng, X. J. and Merigó, J. M. (2015). Framework of group decision making with intuitionistic fuzzy preference information. IEEE Transactions on Fuzzy Systems 23(3), 1211-1227.

[20] Liao, H. C., Xu, Z. S., Zeng, X. J. and Xu, D. L. (2016). An enhanced consensus reaching process in group decision making with intuitionistic fuzzy preference relations. Information Sciences 329, 274-286.

[21] Liu, F., Wu, Y. H. and Pedrycz, W. (2018). A modified consensus model in group decision making with an allocation of information granularity. IEEE Transactions on Fuzzy Systems DOI 10.1109/TFUZZ.2018.2793885.

[22] Liu, J. C. and Li, D. F. (2018). TOPSIS-based nonlinear-programming methodology for multi-attribute decision making with interval-valued intuitionistic fuzzy sets. IEEE Transactions on Fuzzy Systems 26(1), 391-391.

[23] Liu, X. W. and Chen. L. H. (2004). On the properties of parametric geometric OWA operator. International Journal of Approximate Reasoning $35(2), 163-178$

[24] Liu, Y. J., Liang, C. Y., Chiclana, F. and Wu, J. (2017). A trust induced recommendation mechanism for reaching consensus in group decision making. Knowledge-Based Systems 119, 221-231.

[25] Mata, F., Martinez, L. and Herrera-Viedma, E. (2009). An adaptive consensus support model for group decision-making problems in a multigranular fuzzy linguistic context. IEEE Transactions on Fuzzy Systems 17(2), 279-290. 
[26] Mahima, G. (2018). Consensus building process in group decision making-an adaptive procedure based on group dynamics. IEEE Transactions on Fuzzy Systems 26(4), 1923-1933.

[27] Palomares, I. and Martinez, L. (2014). A semisupervised multiagent system model to support consensus-reaching processes. IEEE Transactions on Fuzzy Systems 22(4), 762-777.

[28] Palomares, I., Martinez, L. and Herrera, F. (2014). A consensus model to detect and manage noncooperative behaviors in large-scale group decision making. IEEE Transactions on Fuzzy Systems 22(3), 516530.

[29] Perez, I. J. Cabrerizo, F. J., Alonso, S. and Herrera-Viedma, E. (2014). A new consensus model for group decision making problems with nonhomogeneous experts. IEEE Transactions on Fuzzy Systems Man and Cybernetics Systems 44(4), 494-498.

[30] Perez, I. J., Cabrerizo, F. J., Alonso, S., Dong, Y. C., Chiclana, F. and Herrera-Viedma, E. (2018). On dynamic consensus processes in group decision making problems. Information Sciences 459, 20-35.

[31] Ureña, R., Chiclana, F., Fujita, H and Herrera-Viedma, E. (2015). Confidence-consistency driven group decision making approach with incomplete reciprocal intuitionistic preference relations. KnowledgeBased Systems 89, 86-96.

[32] Victor, P., Cornelis, C., De Cock, M. and Herrera-Viedma, E. (2011). Practical aggregation operators for gradual trust and distrust. Fuzzy Sets and Systems 184(1), 126-147.

[33] Wan, S. P., Wang, F. and Dong, J. Y.(2018). A three-phase method for group decision making with interval-valued intuitionistic fuzzy preference relations, IEEE Transactions on Systems Man CyberneticsSystems, 26(2) 998-1010.

[34] Wang, J. Q., Wang, P., Wang, J., Zhang, H. Y. and Chen, X. H. (2015). Atanassov's interval-valued intuitionistic linguistic multicriteria group decision-making method based on the trapezium cloud model. IEEE Transactions on Fuzzy Systems 23(3), 542-554.

[35] Wu, J. and Chiclana, F.(2012). Non-dominance and attitudinal prioritisation methods for intuitionistic and interval-valued intuitionistic fuzzy preference relations, Expert Systems with Applications, 39 13409-13416.

[36] Wu, J., Sun, Q., Fujita, H. and Chiclana, F. (In press). An attitudinal consensus degree to control feedback mechanism in group decision making with different adjustment cost. Knowledge-Based Systems doi.org/10.1016/j.knosys.2018.10.042 0950-7051

[37] Wu, J., Chang, J. L., Cao, Q. W. and Liang, C. Y. (In press). A trust propagation and collaborative filtering based method for incomplete information in social network group decision making with type-2 linguistic trust. Computers \& Industrial Engineering,doi.org/10.1016/j.cie.2018.11.020

[38] Wu, J., Chiclana, F. and Liao, H. C. (2018). Isomorphic multiplicative transitivity for intuitionistic and interval-valued fuzzy preference relations and its application in deriving their priority vectors. IEEE Transactions on Fuzzy Systems 26(1), 193-202.

[39] Wu, J., Chiclana, F., Fujita, H. and Herrera-Viedma, E. (2017). A visual interaction consensus model for social network group decision making with trust propagation. Knowledge-Based Systems 122, 39-50.

[40] Wu, J., Dai, L. F., Chiclana, F., Fujita, H. and Herrera-Viedma, E. (2018). A minimum adjustment cost feedback mechanism based consensus model for group decision making under social network with distributed linguistic trust. Information Fusion 41, 232-242.

[41] Wu, J., Liu, Y. J. and Liang, C. Y. (2015). A consensus- and harmonybased feedback mechanism for multiple attribute group decision making with correlated intuitionistic fuzzy sets. International Transactions in Operational Research 22(6), 1033-1054.

[42] Wu, Z. B., Ma, N., Zeng, Z. Q. and Xu, J. P. (2018). Integer programming models to manage consensus for uncertain MCGDM based on PSO algorithms. IEEE Transactions on Fuzzy Systems DOI 10.1109/TFUZZ.2018.2866827.

[43] Wu, Z. B. and Xu, J. P. (2018). A consensus model for large-scale group decision making with hesitant fuzzy information and changeable clusters. Information Fusion 41, 217-231.

[44] Xu, Z. S. and Chen, J.(2007). An approach to group decision making based on interval-valued intuitionistic judgment matrices, System Engineer-Theory \& Practice 27, 126-133.

[45] Xu, Y. J., Wen, X. W. and Zhang, W. C. (2018). A two-stage consensus method for large-scale multi-attribute group decision making with an application to earthquake shelter selection. Computers \& Industrial Engineering 116, 113-129.

[46] Yager, R. R. (1993). Families of OWA operators. Fuzzy Sets and Systems $59(2), 125-148$.

[47] Yager, R. R. (1996) Quantifier guided aggregation using OWA operators, International Journal of Intelligent Systems, 11 49-73.
[48] Yager, R. R. and Alajlan, N. (2015) An intelligent interactive approach to group aggregation of subjective probabilities, Knowledge-Based Systems, 83 170-175.

[49] Yan, H. B. and Ma, T. J. (2015). A group decision-making approach to uncertain quality function deployment based on fuzzy preference relation and fuzzy majority. European Journal of Operational Research 241, 815-829.

[50] Yu, W. Y., Zhang, Z., Zhong, Q. Y., and Sun, L. L.(2017). Extended TODIM for multi-criteria group decision making based on unbalanced hesitant fuzzy linguistic term sets, Computers \& Industrial Engineering, 114 316-328.

[51] Zadeh, L. A. (1965). Fuzzy sets. Information and Control 8, 338-353.

[52] Zhang, G. Q., Dong, Y. C. and Xu, Y. F. (2014). Consistency and consensus measures for linguistic preference relations based on distribution assessments. Information Fusion 17, 46-55.

[53] Zhang, H. G., Zhang, J. L., Yang, G. H. and Luo, Y. H. (2015). Leader-based optimal coordination control for the consensus problem of multiagent differential games via fuzzy adaptive dynamic programming. IEEE Transactions on Fuzzy Systems 23(1), 152-163.

[54] Zhang, H. J., Dong, Y. C. and Herrera-Viedma, E. (2018). Consensus building for the heterogeneous large-scale GDM with the individual concerns and satisfactions. IEEE Transactions on Fuzzy Systems 26(2), 884-898.

[55] Zhang, Z., Guo, C. H. and Martínez, L.(2017). Managing Multigranular Linguistic Assignation Assessments in Large-Scale Multiattribute Group Decision Making, IEEE Transactions on Systems Man Cybernetics-Systems, 47 3053-3066.

[56] Zhang, Z., Kuo, X. Y., Yu, W. Y., and Guo, C. H.(2018). On priority weights and consistency for incomplete hesitant fuzzy preference relations, Knowledge-Based Systems, 143 115-126. 